\title{
Cantilever Resin-bonded Bridge Design: A Review
}

\author{
Jenie Rosna Albert ${ }^{1}$, David W Livingstone ${ }^{2}$, Shivasakthy Manivasakan ${ }^{3}$, Varsha Ravichandran ${ }^{4}$
}

\begin{abstract}
Resin-bonded bridges (RBBs) were first described in the 1970s. The present forms of RBBs have evolved from many significant developments. Splinting of periodontally compromised teeth was the initial objective of these prostheses. Their use eventually expanded to replace missing anterior teeth. Cantilever resin-bonded fixed partial denture (RBFPD) is a conservative alternative approach to fixed-fixed partial dentures in replacing missing tooth and should be included as a treatment option wherever possible. For fixed replacement of missing teeth, RBBs can be considered to give a reversible, minimally invasive, esthetic, and predictable restorative outcome in spite of many problems such as debonds. Two-unit cantilevered RBFPDs had a better clinical retention than fixed-fixed RBFPDs because a cantilever RBB eliminates adverse interabutment stresses associated with fixed-fixed designs. The longevity of RBBs is influenced by numerous factors. To achieve successful long-term survival, careful case selection and consideration of various variables like materials used and occlusal protection are crucial.

Keywords: Cantilever, Cantilever resin-bonded bridges, Fixed prosthesis, Resin-bonded bridges, Resin-bonded fixed partial dentures.

Journal of Scientific Dentistry (2020): 10.5005/jp-journals-10083-0925
\end{abstract}

\section{INTRODUCTION}

The present forms of resin-bonded bridges (RBBs) have evolved from many significant developments. RBBs were first described in the 1970s. ${ }^{1}$ Splinting of periodontally compromised teeth was the objective of the prostheses initially. Their use was gradually expanded to replacing of missing anterior teeth in young patients. ${ }^{2}$

Rochette (1973) was the first RBB which is characterized by perforated metal retainer through which the resin cement tag helps in retention. ${ }^{3}$ Thompson and Livaditis developed a technique for the electrolytic etching of cast base metal retainers at the University of Maryland. This is an etched-cast resin-bonded fixed dental prosthesis (RBFDP) that provided micromechanical retention. This design is called as Maryland bridges. More recently, the development of resin cements which bond chemically to both the metal alloy and the tooth surface has enhanced the retention of bridge. ${ }^{3}$

The main advantage of RBBs is that with conservative abutment preparation, it provides a fixed replacement of missing teeth. This gives the possibility of reduction of the risk of endodontic complications in abutment teeth. The success of these resin-bonded fixed partial dentures (RBFPDs) depends on bonding between the metal casting and etched enamel, and precise and defined metal engagement of the abutment is required. Clinical studies now show that for the replacement of short spans over short to medium term, RBFPDs have a predictable restorative option with the development of tooth preparation, prosthesis design, and adhesive resin cements. ${ }^{4}$

The longevity of this type of bridgework is the main concern since its introduction. Many authors have reviewed the clinical performance of RBBs with a range of success reported. Failure rate as low as $11.5 \%$ in anterior and as high as $83.3 \%$ in the posterior was reported. ${ }^{5}$ In a study conducted for the replacement of single tooth, cantilever RBB design showed the lowest debond rate. ${ }^{6}$ As reported by another retrospective study, the cantilever RBBs were as successful as fixed-fixed type. An effective means of managing partially debonded RBBs is the conversion of rebonded retainer into cantilever RBBs. ${ }^{5}$
${ }^{1-4}$ Department of Prosthodontics and Crown and Bridge, Indira Gandhi Institute of Dental Sciences, Sri Balaji Vidyapeeth, Puducherry, India

Corresponding Author: Jenie Rosna Albert, Department of Prosthodontics and Crown and Bridge, Indira Gandhi Institute of Dental Sciences, Sri Balaji Vidyapeeth, Puducherry, India, Phone: +91 9597480721, e-mail: jenierosna@gmail.com

How to cite this article: Albert JR, Livingstone DW, Manivasakan S, Ravichandran V. Cantilever Resin-bonded Bridge Design: A Review. J Sci Den 2020;10(1):28-30.

Source of support: Nil

Conflict of interest: None

\section{Advantages}

Conservative tooth preparation; reversible as the bridges can be removed with minimal damage to abutment, and thus, it can be used as interim restoration; cost effective; less time consuming and patient satisfaction due to lack of tooth preparation; reduced chair time and cost. ${ }^{7}$

\section{Disadvantages}

Failure due to debond because of poor bridge design and cementation technique is the most common factor in RBBs. RBBs are technique sensitive and compromised esthetic which can cause incisal shine-through of metal if an opaque cement is not used. ${ }^{7}$

\section{Cantilever RBB Design}

It has been widely reported that RBBs are more successful as cantilever RBBs than as fixed-fixed restorations. The fixed-fixed or RBB with multiple abutments debonded because of differential movement of abutment teeth, and this was more evident when there was natural tooth surface on the occlusal contact. In these cases, the lute cement failed as the occlusal force leads the tooth and the retainer to be driven apart. ${ }^{3}$ In a study conducted, there was no debond of cantilever bridge design during the evaluation period when compared to fixed-fixed design. ${ }^{5}$ Two-unit cantilevered 
RBFPDs had a better clinical retention than fixed-fixed RBFPDs because a cantilever RBB eliminates adverse interabutment stresses associated with fixed-fixed designs. ${ }^{8-10}$

\section{Advantages of Cantilever RBBs}

The advantages of cantilever RBB include conservative tooth preparation, rational technique used for fabrication, realization of retention loss, improved and simplified oral hygiene as the dental floss could be passed through the open proximal contact area, and it is also possible to create diastema if esthetic is of concern in case of too wide edentulous area. ${ }^{11}$

\section{Case Selection}

The patient-related parameters include various factors such as age of the patient, patient's expectation, location of the pontic, assessment of the abutment tooth, and occlusion. ${ }^{12}$

The abutment tooth selection was done based on the adequate clinical height of the crown, periodontal health of the tooth, minimally restored tooth, and the availability of enamel on the abutment tooth. ${ }^{12}$

Other considerations include the coverage of retainer, preparation of tooth, selection of alloy, use of grooves, and pontic form. $^{12}$

Debonding of RBB, porcelain fracture, caries on the abutment tooth, root resorption, loss of abutment due to periodontitis, framework fracture, retainer cusp fracture, and minor rotation lead to complication in RBBs. ${ }^{12}$

\section{Tooth Preparation}

RBBs can be done without or with minimal tooth preparation. Bond strength in enamel is better than that of dentine. A modified tooth preparation would enhance the retention and resistance form of RBBs. Distinct proximal grooves and a labial extension were the most effective preparations. ${ }^{13}$

\section{Modified Tooth Preparation}

These include creating a defined path of insertion, extending the framework to the maximum on the lingual aspect of the abutment teeth, defined cingulum and occlusal rest preparations, and proximal grooves for posterior RBBs. ${ }^{14}$

\section{Retainer Type}

There is a wide range of retainer type used for RBBs which varies from the perforated prototype to the chemically etched retainers that are used at present. ${ }^{14}$ The proximal preparation design and retainer type had no significant effect on survival of RBBs. ${ }^{15}$

\section{Occlusion and RBBs}

The pontic should be in light contact in intercuspal position in order to control the axial position of the opposing tooth, but not involved in guidance. When a cantilever design is used, the guidance should be shared with the natural teeth if the guidance on the pontic cannot be avoided. ${ }^{16}$

\section{Cementation}

The RBFDP restoration survival seems to rely on the resin bond and not on any additional mechanical retention. ${ }^{17}$ Degradation and reduced bond strength with time were exhibited by composite resin material. In contrast, due to the formation of a chemical bond between the phosphate group of the cement monomer and the oxide layer of the metal retainer, prolonged high bond strengths were demonstrated by Panavia (Karrary Co. Ltd, Osaka, Japan). To enhance further retention, micromechanical interlocking created by sandblasting should be carried out before cementation. ${ }^{2}$ It has been reported that micromechanical retention was better than macromechanical retention. ${ }^{18}$

\section{Survival Rate of Material Used}

Excellent long-term survival rate of $91 \%$ after 10 years and $84 \%$ after 15 years has been reported for metal-ceramic cantilever RBFPDs. ${ }^{19}$ The survival rate for all-ceramic cantilever RBFPDs for both 10 years and 15 years was $95.4 \%$ and reduced to $81.8 \%$ after 18 years. ${ }^{19}$ Cantilever all-ceramic RBFPDs gave a better outcome than metal-ceramic cantilever RBFPDs for the replacement of missing anteriors. ${ }^{20}$ Zirconia-ceramic RBFDPs yielded a 10 -year survival rate of $98.2 \%$ and a success rate of $92.0 \%$; thus, excellent clinical longevity was provided by anterior zirconia-ceramic cantilever RBFDPs. ${ }^{21}$ The glass-ceramic RBFPDs had a 6 -year survival rate of $100 \%$ that exhibits an excellent clinical performance for anterior and posterior regions. ${ }^{22}$ For a long-term durable bonding to zirconia ceramic under humid and stressful oral conditions, a combined use of adhesive resin containing 10-methacryloyloxydecyl dihydrogen phosphate and air abrasion at a moderate pressure can be used. Occlusal protection and technical protocol are crucial. ${ }^{23}$ The fracture and debonding rate of anterior all-ceramic cantilever RBFDPs can be minimized with the use of phosphate monomers containing resin cements and zirconia ceramic. ${ }^{24}$

\section{Summary}

Though for many decades RBFPDs have been used, it had suffered from some disadvantages, often related to frequent debonding, decays under the abutment teeth, use of metal for the infrastructure, and retainer which was unesthetic. This is often due to a misunderstanding about the tooth preparation design, choice of material, retainer design, choice of abutment tooth, and choice of adhesive system. ${ }^{1}$ Occlusal protection and technical protocol are crucial for long-term survival of the fixed dental prosthesis. ${ }^{23}$ The replacement of missing teeth with cantilever RBFPDs is a conservative alternative to conventional fixed partial dentures and should be included as a treatment option. For fixed replacement of missing teeth, RBBs can be considered to give a reversible, minimally invasive, esthetic, and predictable restorative outcome in spite of many problems such as debonds. Thus, careful patient selection, treatment planning, and attention to all factors will help to fabricate successful restorations with longer survival rates.

\section{References}

1. Sillam CE, Cetik S, Ha TH, Atash R. Influence of the amount of tooth surface preparation on the shear bond strength of zirconia cantilever single-retainer resin-bonded fixed partial denture. J Adv Prosthodont 2018;10(4):286-290. DOI: 10.4047/jap.2018.10.4.286.

2. Alraheam IA, Ngoc CN, Wiesen CA, Donovan TE. Five-year success rate of resin-bonded fixed partial dentures: a systematic review. J Esthet Restor Dent 2019;31(1):40-50. DOI: 10.1111/jerd.12431.

3. Durey KA, Nixon PJ, Robinson S, Chan MY. Resin bonded bridges: techniques for success. Br Dent J 2011;211(3):113. DOI: 10.1038/ sj.bdj.2011.619.

4. Botelho MG, Ma X, Cheung GJ, Law RK, Tai MT, Lam WY. Long-term clinical evaluation of 211 two-unit cantilevered resin-bonded fixed partial dentures. J Dent 2014;42(7):778-784. DOI: 10.1016/j. jdent.2014.02.004. 
5. Chan AW, Barnes IE. A prospective study of cantilever resin-bonded bridges: an initial report. Aust Dent J 2000;45(1):31-36. DOI: 10.1111/ j.1834-7819.2000.tb00239.x.

6. Dunne SM, Millar BJ. A longitudinal study of the clinical performance of resin bonded bridges and splints. Br Dent J 1993;174(11):405-411. DOI: $10.1038 /$ sj.bdj.4808186.

7. St George G, Hemmings K, Patel K. Resin-retained bridges re-visited part 1. history and indications. Prim Dent Care 2002;9(3):87-91. DOI: 10.1308/135576102322492927.

8. Wong TL, Botelho MG. The fatigue bond strength of fixed-fixed versus cantilever resin-bonded partial fixed dental prostheses. J Prosthet Dent 2014;111(2):136-141. DOI: 10.1016/j.prosdent.2013.07.004.

9. Hussey DL, Linden GJ. The clinical performance of cantilevered resinbonded bridgework. J Dent 1996;24(4):251-256. DOI: 10.1016/03005712(95)00073-9.

10. Tezulas E, Yildiz C, Evren B, Ozkan Y. Clinical procedures, designs, and survival rates of all-ceramic resin-bonded fixed dental prostheses in the anterior region: a systematic review. J Esthet Restor Dent 2018;30(4):307-318. DOI: 10.1111/jerd.12389.

11. Kern M. Two-joint etched bridges. Quintessence Int 2018;49:789-798.

12. Abuzar M, Locke J, Burt G, Clausen G, Escobar K. Longevity of anterior resin-bonded bridges: survival rates of two tooth preparation designs. Aust Dent J 2018;63(3):279-284. DOI: 10.1111/adj. 12612

13. Burgess JO, McCartney JG. Anterior retainer design for resin-bonded acid-etched fixed partial dentures. J Prosthet Dent 1989;61(4): 433-436. DOI: 10.1016/0022-3913(89)90009-7.

14. Balasubramaniam GR. Predictability of resin bonded bridges-a systematic review. Br Dent J 2017;222(11):849. DOI: 10.1038/ sj.bdj.2017.497.

15. Cötert HS, Öztürk B. Posterior bridges retained by resin-bonded cast metal inlay retainers: a report of 60 cases followed for 6 years. J Oral Rehabil 1997;24(9):697-704. DOI: 10.1046/j.1365-2842.1997. 00548.x.
16. Gulati JS, Tabiat-Pour S, Watkins S, Banerjee A. Resin-bonded bridgesthe problem or the solution? Part 1: assessment and design. Dent Update 2016;43(6):506-521. DOI: 10.12968/denu.2016.43.6.506.

17. Wei YR, Wang XD, Zhang Q, Li XX, Blatz MB, Jian YT, et al. Clinical performance of anterior resin-bonded fixed dental prostheses with different framework designs: a systematic review and meta-analysis. J Dent 2016;47:1-7. DOI: 10.1016/j.jdent.2016.02.003.

18. Creugers $\mathrm{NH}$, Käyser AF. An analysis of multiple failures of resinbonded bridges. J Dent 1992;20(6):348-351. DOI: 10.1016/03005712(92)90023-6.

19. Kern M. Fifteen-year survival of anterior all-ceramic cantilever resin-bonded fixed dental prostheses. J Dent 2017;56:133-135. DOI: 10.1016/j.jdent.2016.11.003.

20. Saker S, El-Fallal A, Abo-Madina M, Ghazy M, Özcan M. Clinical survival of anterior metal-ceramic and all-ceramic cantilever resin-bonded fixed dental prostheses over a period of 60 months. Int J Prosthod 2014;27(5):422-424. DOI: 10.11607/ijp.3776.

21. Kern M, Passia N, Sasse M, Yazigi C. Ten-year outcome of zirconia ceramic cantilever resin-bonded fixed dental prostheses and the influence of the reasons for missing incisors. J Dent 2017;65:51-55. DOI: 10.1016/j.jdent.2017.07.003.

22. Sailer I, Bonani T, Brodbeck U, Hans Franz Hämmerle C. Retrospective clinical study of single-retainer cantilever anterior and posterior glass-ceramic resin-bonded fixed dental prostheses at a mean follow-up of 6 years. Int J Prosthod 2013;26(5):443-450. DOI: 10.11607/ ijp.3368.

23. Klink A, Huettig F. Zirconia-based anterior resin-bonded singleretainer cantilever fixed dental prostheses: a 15-to 61-month follow-up. Int J Prosthodont 2016;29(3):284-286. DOI: 10.11607/ ijp.4220.

24. Mourshed B, Samran A, Alfagih A, Samran A, Abdulrab S, Kern M. Anterior cantilever resin-bonded fixed dental prostheses: a review of the literature. J Prosthodont 2018;27(3):266-275. DOI: 10.1111/ jopr.12555. 